\title{
THE PRESENT EUROPEAN ICHTHYOHAEMATOLOGY AND PROSPECTS TOWARDS THE 21ST CENTURY
}

\author{
D. PRAVDA
}

University of Veterinary and Pharmacological Sciences, Brno, Czech Republic

The scientific conference, at which we have just been welcomed by its kind organizers, the representatives of Czech university scientific institutions, namely the South Bohemian Agricultural University Ceské Budějovice and the University of Veterinary and Pharmaceutical Sciences Brno, is chronologically already the fifth, thus being a sort of jubilee ichthyohaematological conference. The remarkably high frequency of these professional meetings - from the first conference of this type which had world priority in the year 1985 "only" 13 years elapsed. This fact eloquently documents the extraordinary scientific fruitfulness of all their participants and at the same time shows the dynamic trend in the constitution and development of a young branch of haematology.

The very beginnings of European ichthyohaematology must be sought in the sporadic scientific communications of zoologists with the basic outline findings employed and cited to this very day, especially in studies of many aspects of comparative animal haematology (total blood counts of fish, blood coagulation, morphology of nucleated erythrocytes, morphological specificities of fish blood, etc.). These papers cover the period of the 1940s and 1950s (for example, studies by Toth 1948, Steffens 1955, Antipova 1954, Kudryavtseva 1961 and others).

Let me mention five basic factors. the main catalyzers of the moulding of ichthyohaematology into the form which we have known during past 13 years.

The first factor was the stormy postwar development of human and animal haematology, especially the lesson of the applicability of haematological findings in the diagnostics of numerous animal diseases (e.g. young animal anaemias, avian and mammalian leukoses) which lead to the great developments of laboratory techniques, such as the application of haematological micromethods, and standardization of haematological procedures.

The second factor was the postwar transformation of the European freshwater fishery, characterized by new intensive forms of fish breeding, aimed especially at a high fish production and the ensuing health problems in fish.

The third factor with a catalyzing influence on the development of ichthyohaematology was the rapid postwar development of industry, especially the chemical and petrochemical industries, linked with hitherto almost unknown abnormal ecological burden to waters, including production waters and the adverse effects on fish health.

The fourth factor was the excellent communication between specialists in fish haematology that was so helpful in creating (beside numerous and valuable collegial friendships) the necessary conditions for wide international cooperation that culminated in the abovementioned four scientific meetings. All these conferences yielded an important literature enrichment including more than 150 scientific papers. This collection represents today the largest and most complex proceedings in European ichthyohaematology.

Last but not least, the fifth factor stimulating the European ichthyohaematology of present days are the remarkable organizational activities, especially those of the Research Institute of Fish Culture and Hydrobiology in Vodñany. This Institute has literally become the driving force and engine of such activities. It is my great pleasure to mention at least the pioneer scientific work of Assistant Professor Dr. Z. Svobodová, DrSc. and her colleagues. Through the importance and number of her publications, high scientific standard and conceptuality this dear colleague of ours has rightly become - and you will all certainly agree with me - an important protagonist of this branch of haematology. 
Now as regards the current state and standard of our findings and experience in ichthyohaematology. I would like to accentuate four points:

First, pioneer findings in physiological blood indices of more than 20 freshwater species under predominantly defined breeding and life conditions of fish in often non-equivalent European regions. The scope of these findings was widened last year by fish population haematology presented in one of hitherto most extensive works (Lusková 1997); we shall certainly hear more about it at this Conference. These results of population ichthyohaematology became a valuable contribution to general haematology.

Secondly, the present ichthyohaematology provides practical applications worked out for fishery, veterinary and diagnostic purposes, and especially for practical ecology. Ichthyohaematology thus contributes to needs of society, lately also by numerous and important papers in ichthyotoxicology and ichthyoimmunology. Many of us who still remember these ichthyohaematological meetings recall that already at the Second International Fish Blood Conference in 1989 a section on fish immunology was included. Then a prudent prognosis has today become a reality. Thanks to ichthyohaematology we have ascertained that environmental defects. ecological hazards or various dysbalances lead in their consequences to damage to the immune system, and health impairment of the fish. Ichthyohaematology has thus become a significant tool in the ecological analysis of the environment. Papers that will now be presented bring an important benefit in ecological haematology.

Third, there is a relatively high standard of the existing experimental fish haematology with great progress in modelling of the environment of fish and their haematological, biochemical, hormonal and immunological responses.

Fourth, there is the high standard of international information exchange in the form of lectures (Lebedeva 1993, Schwaiger 1997) and study visits (Kreutzmann 1985).

Let me finally take the liberty to mention another, new aspect of ichthyohaematology, i.e. its effective implementation in the university course named "Veterinary Haematology". It is my responsibility in cooperation with Prof. Smetana, Chair of Human Haematology in the Baylor Medical Centre, Houston, Texas, USA to teach this subject to students of the 4th and 5th year of veterinary medicine, veterinary hygiene and ecology and also for students of the Natural Sciences, Faculty of the Masaryk University and the Mendel University of Agriculture and Forestry. This course has already been taking place for three years. Findings in ichthyohaematology have a prominent place in the relevant section on comparative haematology. Thanks to our accentuation of the evolution of blood formation and the development of blood elements, ichthyohaematology plays a very useful role in understanding the interconnections of the evolution development stages between animal classes.

I truly believe that in this young branch of haematology (younger is only the herpetohaematology just being constituted), we have attained good success and findings of high importance and in their consequences reach beyond fishery itself. The initiated conception has proved to be fully justified and therefore we need to continue along this road. The innovation processes presume methodologies of ichthyohaematological research, including the introduction of a central information network. Cooperation should be offered to all other European countries. Large potentials remain in cooperation with Japan and the United States. This, however, would include a wider approach and include research of salt-water fishes. I believe that this, too, is a realistic perspective. The interconnection of ichthyohaematology and ecology, and perhaps you will agree, I would consider to be a fundamental evolutionary feature and therefore also the main task for the future research and international cooperation. For this endeavour I wish you and your colleagues at home all the success, good health and a creative atmosphere. I also wish you inspiring discussions at this conference. 